\section{The role of association between the cholinergic precursor choline alphoscerate and sulodexide in vascular dementia}

\author{
Angelo Di Salvo \\ Simple Long-Term Care Unit, Bianchi's \\ Hospital of Corleone, ASP Palermo, \\ Italy
}

\begin{abstract}
Dementia is a syndrome of acquired intellectual deficit resulting in significant impairment of social or occupational functioning. Vascular dementia $(\mathrm{VaD})$ is the second most common causes of dementia after Alzheimer's disease, causing around 15\% of cases. However, unlike Alzheimer's disease an involvement of the cerebral cholinergic system in the pathophysiology of $\mathrm{VaD}$ has been hypothesized and there is no standard treatment. In the Vascular Dementia Italian Sulodexide Study (VA.D.I.S.S.) the positive results obtained with the sulodexide are worthy of note. In this study 40 elderly subjects with recent onset (less than 9 month) slight to moderate mental deterioration due to vascular origin were observed for nine months during oral treatment with sulodexide and choline alphoscerate with the aim of analyzing whether therapeutic effects can be enhanced. These preliminary results suggest that the additional therapy of choline alphoscerate with sulodexide represents a way to increase the beneficial effects of cholinergic therapies in the $\mathrm{VaD}$ and improve all the different examinate score: mini mental state evaluation (MMSE), basic activities of daily living (ADL), instrumental activities of daily living (IADL).
\end{abstract}

\section{Introduction}

Vascular dementia (VaD), a heterogeneous group of brain disorders, defines alterations in cognition, ranging from minor deficits to full-blown dementia. ${ }^{1-5}$ Attributable to cerebrovascular causes is responsible for at least $15 \%$ of cases of dementia, being second only to Alzheimer's disease (AD). ${ }^{6}$ The cognitive outcome of patients with VaD may be as severe as in $\mathrm{AD}$, but morbidity and mortality are usually worse. The conventional vascular risk factors (arterial hypertension, diabetes, atherosclerosis, smoking, etc.) explain only par- tially the causes of vascular dementia. ${ }^{7-9}$ The diagnosis of $\mathrm{VaD}$ resides in clinical criteria determining a cognitive impairment, ${ }^{10}$ the presence of cerebrovascular disease and, only in the case of post-stroke dementia or multi-infarct dementia, a temporal relationship between these. Central to the disease mechanism is the crucial role that cerebral blood vessels play in brain health, ${ }^{11,12}$ not only for the delivery of oxygen and nutrients, but also for the trophic signaling that links inextricably the well-being of neurons and glia to that of cerebrovascular cells. ${ }^{13}$ Cholinergic deficits are well documented in $\mathrm{VaD}$, independently of any concomitant alterations. ${ }^{14}$ Cholinergic structures are vulnerable to ischemic damage particularly of the telencephalon as well as numerous brain areas involved in cognitive activities..$^{15}$ Vascular disorders can worsen the performance of daily activities causing frequent requests for intervention by health facilities $^{16,17}$ Based on these considerations, in our study two active drugs were used, one on vascular problems and the other on the production of acetylcholine and both on cholinergic hypofunction.

\section{Choline alphoscerate in vascular dementia}

Choline alphoscerate (alfa glycerylphosphorylcholine) is a precursor of second generation choline. From the biochemical point of view it is a phospholipid: in fact it enters the composition of cell membranes. ${ }^{18}$ It easily crosses the blood-brain barrier after oral intake and, when it reaches the brain, increase the brain content of choline and the release of acetylcholine because choline alphoscerate is hydrolyzed to choline which is the precursor of the neurotransmitter acetylcholine, and is used for the improvement of cognitive dysfunction in patients with dementia of neurodegenerative and vascular origin. ${ }^{19-21}$ Some of its specific activities: better ATPase activity of membranes, anti-edema effect on the neuron, during cerebral ischemia. It would antagonize the increase in NEFA induced by cerebral ischemia, stimulate the biosynthesis of phospholipids of membranes during cerebral hypoxia. It reduces the excitation threshold values of the pyramidal system. It would have dopaminergic activity by acting on the nerve centers which depend on the substantia nigra. It has an elimination halflife of 0.5-6.2 hours and is completely absorbed following oral administration. The pharmacokinetic studies demonstrate the excellent bioavailability of the oral formulation which is indicated in cerebrovascular pathology. ${ }^{22}$ It is estimated that it will be incorporated into brain phospholipids within 24 hours of absorption. Furthermore, the
Correspondence: Angelo Di Salvo, via Luca Giordano 2, 90011 Bagheria (PA), Italy E-mail: angelo.disalvodoc@gmail.com

Key words: Sulodexide; choline alphoscerate vascular dementia; small vessel disease.

Conflict of interest: the author declares no potential conflict of interest.

Availability of data and materials: the data used to support the findings of this study are available from the corresponding author upon request.

Ethics approval and consent to participate: being an observational study was only notified to the local ethics committee. All participants in the study were informed about the aims of the research before enlisting them and their privacy was protected throughout the course of the study.

Received for publication: 2 June 2021.

Revision received: 2 January 2022.

Accepted for publication: 12 January 2022

This work is licensed under a Creative Commons Attribution-NonCommercial 4.0 International License (CC BY-NC 4.0).

${ }^{\circ}$ Copyright: the Author(s), 2021

Licensee PAGEPress, Italy

Geriatric Care 2021; 7:9891

doi:10.4081/gc.2021.9891

increase in brain levels of choline after oral intake occurs significantly more than in another choline precursor, cytidine-5diphosphocoline. ${ }^{23}$ Choline alphoscerate stimulates the release of acetylcholine in the hippocampus and striatum, improving the learning memory processes. Choline alphoscerate has been shown to improve mnesic and attentional performance in humans with cognitive vascular disorder with greater efficacy than Lacetylcarnitine. ${ }^{24}$ Similarly, the increase in memory and attention scores, and an improvement in affective and somatic symptoms was observed in three other homogeneous studies on patients with cerebral vascular insufficiency. ${ }^{25,26}$

\section{Sulodexide in vascular dementia}

Sulodexide (SDX), a sulfated polysaccharide complex extracted from porcine intestinal mucosa, is a blend of two glycosaminoglycan (GAG) entities, namely a fast-moving heparin (HP) fraction and a dermatan sulfate (DS: 20\%) component. The compound is unique among HP-like substances in that it is biologically active by both the parental and oral routes. SDX administrated parenterally displays an 
antithrombotic action like that of HPs but associated with other alterations of the blood clotting mechanism and tests, thus being much less conducive to bleeding risk than HPs. When given orally, SDX is associated with minimal changes in classic coagulation tests but maintains several important effects on the structure and function of endothelial cells (EC), and the intercellular matrix. These activities include prevention or restoration of the integrity and permeability of $\mathrm{EC}$, co-interaction versus chemical, toxic or metabolic EC injury, regulation of EC-blood cell interactions, inhibition of microvascular inflammatory and proliferative changes, and other similar effects, thus allowing oral SDX to be considered as an endothelial-protecting agent. ${ }^{27}$ In the VADISS (VaD Italian SDX study), a multicenter, double-blind trial, 86 patients with ascertained $\mathrm{VaD}$ were randomly treated with oral SDX or PTX for 6 months, efficacy assessed by means of a validated rating scale for dementia (GBS) was in favor of SDX $v s$ PTX in terms of the amelioration of motor, cognitive, and emotional functions.

\section{Materials and Methods}

Aim of the study was to verify the efficacy and tolerability of an oral formulation of choline alfoscerate plus sulodexide in elderly patients with vascular dementia.

The study was not sponsored by pharmaceutical companies that manufactured the tested drugs. The medicinal products used were prescribed at the dosages recommended in the marketing authorization. The inclusion of patients in each therapeutic strategy was not decided in advance but at random and the decision to prescribe the drug was entirely independent of the decision to include the patient in the study. Patients were informed about participation in the study and their privacy was protected throughout the course of the study. Being an observational study was only notified to the local Ethics Committee.

\section{Subjects}

The observational study included 40 elderly ( 22 females, 18 males, mean age 77 $+/-8$, range 69-85) enrolled from ambulatory of Geriatrics of the Hospital 'dei Bianchi of Corleone'. All patients also need to have ischemic brain lesion documented by neuroimaging (MRI and/or CT scan) ${ }^{28}$ with a score $>2$ according to the new rating scale for age-related white matter changes (ARWMC) and NINDS-AIREN diagnostic criteria for probable vascular dementia, and a mini mental state examination (MMSE) score between 11 and 22 was used as inclusion criteria.

The inclusion criteria in the study were: MMSE between 22 and 11; score $>2$ on the ARWMC, the scale for evaluating ischemic brain damage assessed with computed tomography and/or magnetic resonance imaging of the brain, presence of at least two of the following vascular risk factors: arterial hypertension, diabetes, obesity, ischemic heart disease, dyslipidemia, hyperhomocysteinemia, smoking, previous cerebrovascular events and/or cardio-cerebrovascular diseases.

The exclusion criteria were: decompensated heart disease; severe hepatic and/or renal insufficiency; uncorrected dysthyroidism; evolutionary systemic pathologies (e.g., neoplasms); diagnosis of major depression according to DSM-IV criteria); concomitant treatment with NAO or warfarin; coagulation disorders.

Eligible patients were divided into two groups of 20 people: i) treatment with sulodexide $200 \mathrm{mg}$ twice a day + choline alphoscerate $600 \mathrm{mg}$ per day; ii) treatment only with alphoscerate choline $600 \mathrm{mg}$ per day. Table 1 shows the characteristics of the enrolled patients.

\section{Outcome assessment and statistical analysis}

Patients were evaluated at baseline (T0), at three months (T1) and after 9 months (T2 6 months after T1). The main outcomes sought were: MMSE, activities of daily living (ADL) and instrumental activi-

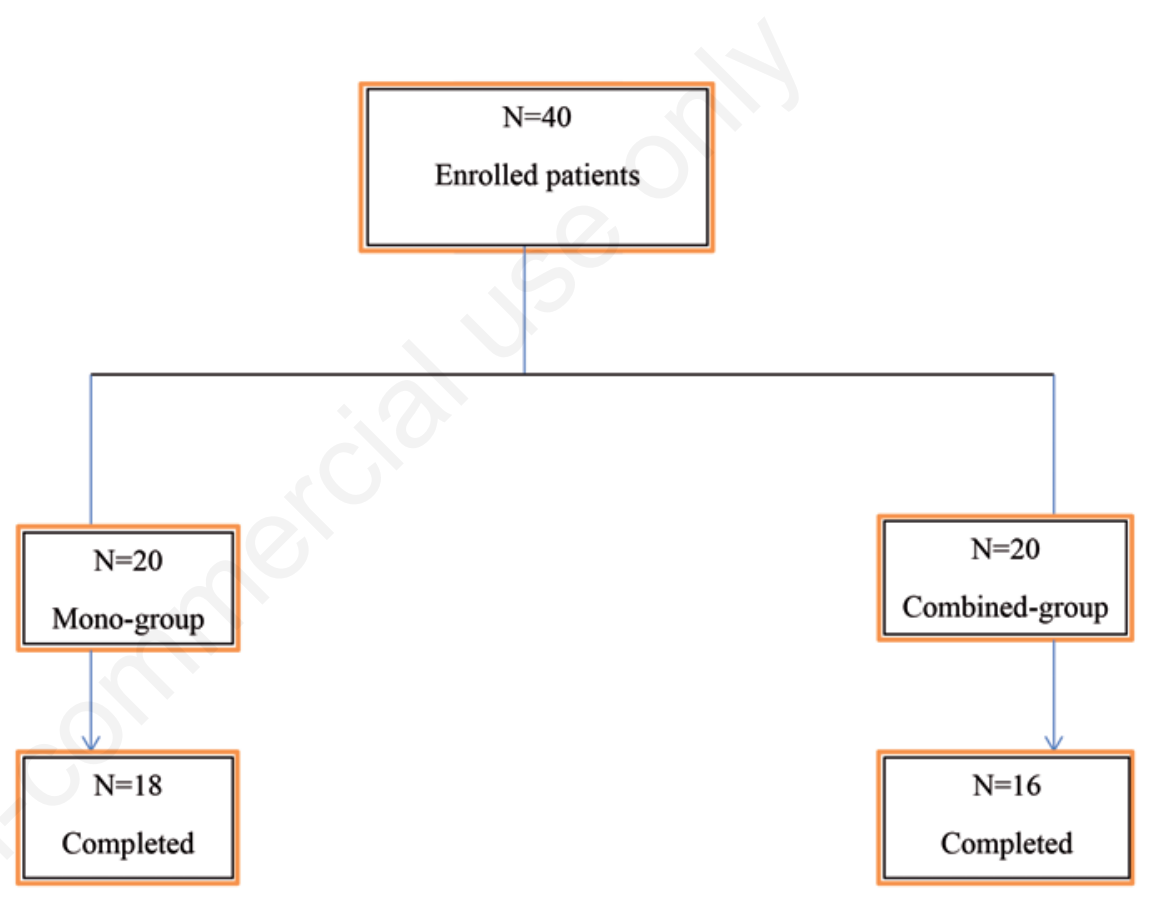

Figure 1. Disposition of subjects in two groups.

Table 1. Demographic characteristics and medical history of patients with vascular dementia enrolled in the study.

\begin{tabular}{lcc} 
Variables & Monotherapy (20) & Active treatment (20) \\
Male, $n(\%)$ & $9(45 \%)$ & $9(45 \%)$ \\
Female, $n(\%)$ & $11(55 \%)$ & $11(55 \%)$ \\
\hline Age (years) mean (SD) & $76+/-6$ & $78+/-7$ \\
Cardiovascular disease, $n(\%)$ & $8(40 \%)$ & $9(45 \%)$ \\
\hline Myocardial infarction, n (\%) & $3(15 \%)$ & $2(10 \%)$ \\
Hypertension, n (\%) & $20(100 \%)$ & $20(100 \%)$ \\
\hline Diabetes mellitus, n(\%) & $6(30 \%)$ & $6(30 \%)$ \\
MMSE, mean (SD) T0 & $21.6+/-0.4$ & $20.8+0.4$ \\
\hline ADL, mean (SD) T0 & $2+/-0.3$ & $2+/-0.4$ \\
IADL, mean (SD) T0 & $2+/-0.2$ & $3+/-0.5$ \\
\hline
\end{tabular}

MMSE, mini mental examination; ADL, activities daily living; IADL, instrumental activities daily living. 
ties of daily living (IADL) (significance $<0.05)$ vs the control group. Adverse effects were also investigated.

Data are presented as mean and standard deviation from continuous variables. All data were using analyzed descriptive statistical analysis.

The results of each follow-up were compared with the initial ones in a functional cognitive battery.

Statistical comparisons between different time points were performed using the ANOVA test for repeated measures, with a $\mathrm{P}$ value $<0.05$ considered statistically significant.

\section{Results}

Among the 40 subjects who were enrolled six subjects (two receiving mono and four receiving combined therapy) no return on time before of $\mathrm{T} 1$ assessment (three months past enrollment) and were therefore excluded from the study (Figure 1). The reasons for the withdrawal were not related to the two drugs taken in this study. In particular, two patients (one in the monotherapy group and the other in the combined therapy group died), the other four patients did not continue the study for cardiovascular problems (three for the onset of atrial fibrillation with need of anticoagulant therapy and one for hospitalization due to heart failure).

As can be seen, already in the first three months of the study, it is possible to appreciate an improvement in the scores of MMSE in patients treated with the combination of sulodexide and choline alphoscerate compared to those treated with monotherapy. At nine months, the improvement in the active treatment group was significant $(\mathrm{P}<0.05)$ compared to the standard treatment. The MMSE trend is summarized in Figure 2.

On the contrary, the association between choline alphoscerate and sulodexide shows a slight improvement in ADL and IADL values only after nine months of treatment (ADL: +1.5 ; IADL: $+1 ; \mathrm{P}<0.05$ ). This result is likely to be explained by the fact that vascular dementia causes more significant worsening on executive functions rather than on memory ones. Figure 3 in panels A and B summarizes the ADL and IADL data.

No available adverse events were reported among all two groups.

The results suggested that taking for a least nine months of the combination therapy of choline alphoscerate plus SDX has more effects than the administering of choline alphoscerate alone on patients with $\mathrm{VaD}$.

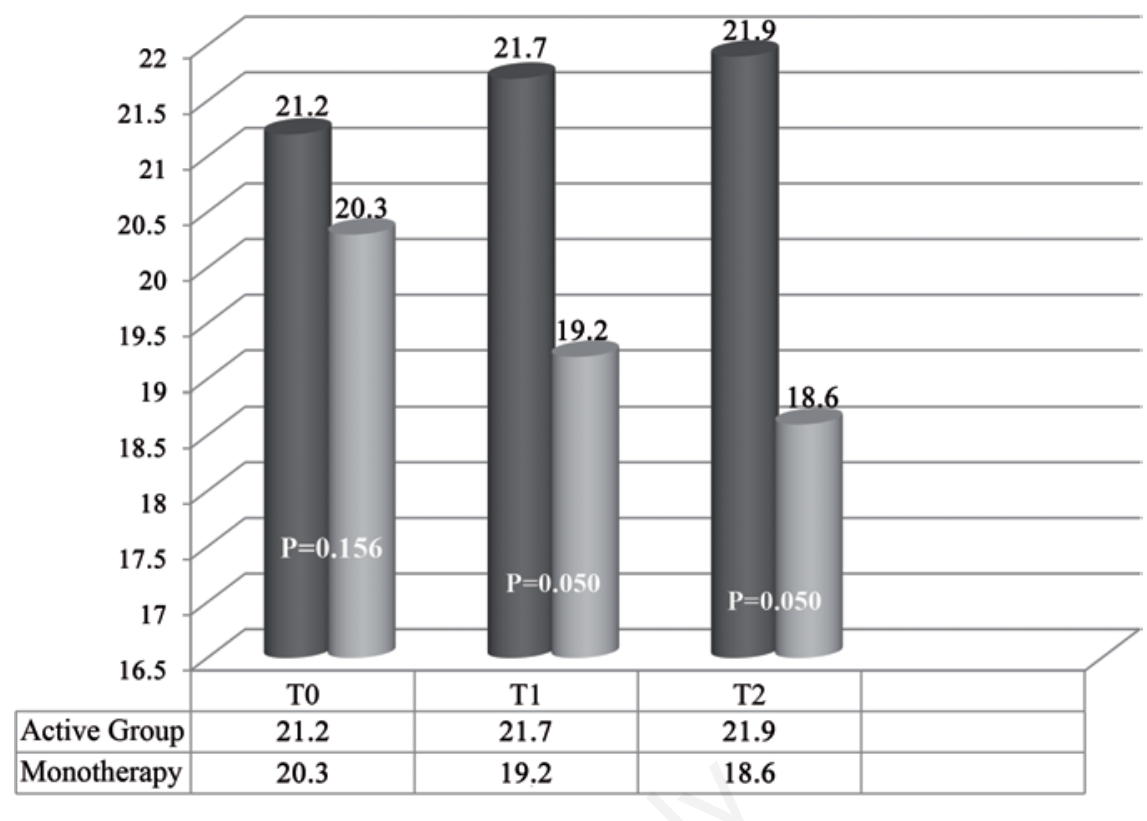

Figure 2. Trend of mini mental examination scores during the study.

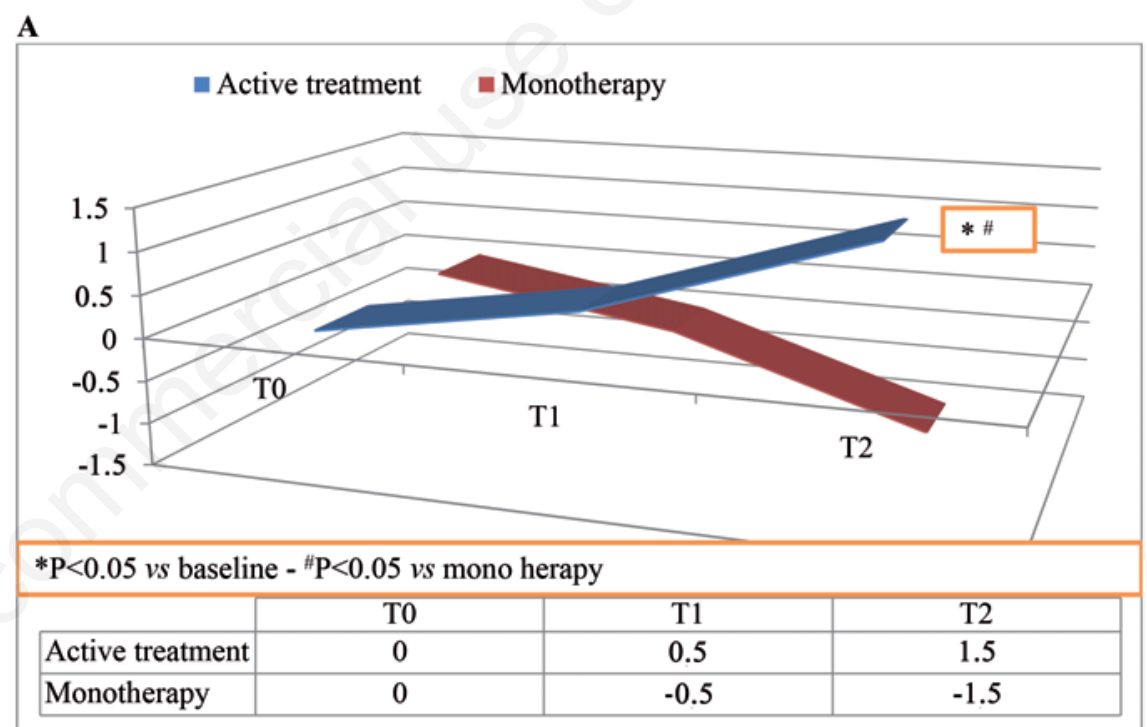

B

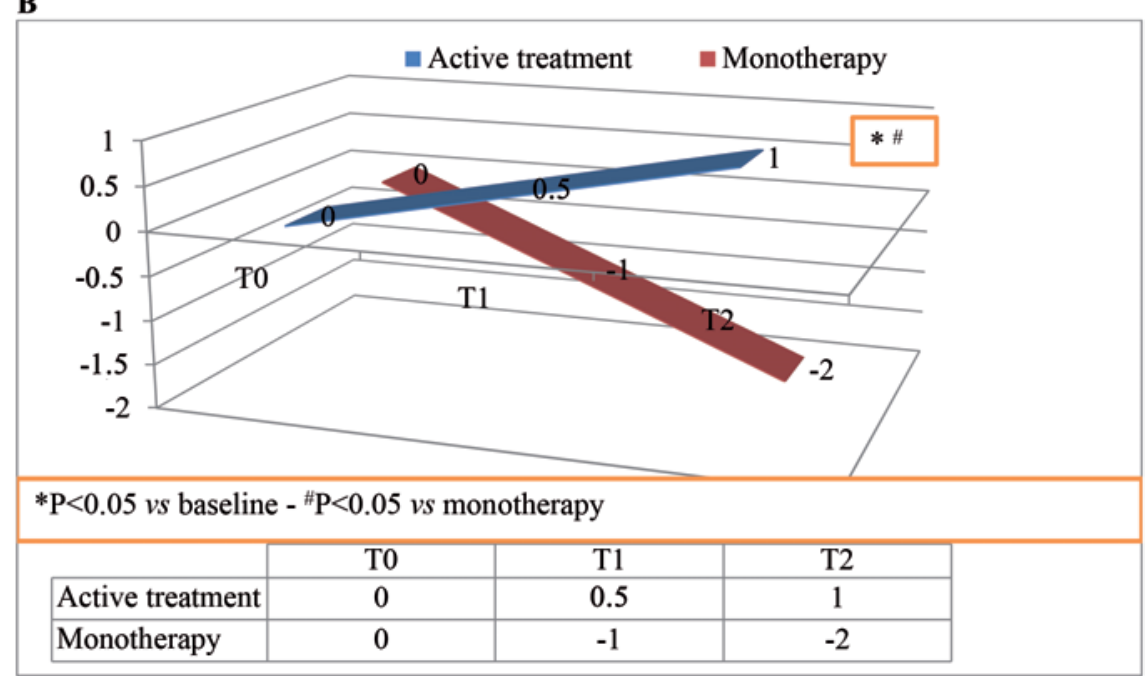

Figure 3. A) Trend of activities daily living; B) trend of instrumental activities daily living. 


\section{Discussion}

Vascular dementia is a very common cause of dementia sindromes. ${ }^{29}$ Few therapeutic options are currently available to improve the prognosis of patients with $\mathrm{VaD}$ and there is an urgent need for interventional studies in this condition. An effective therapy for VaD patients' needs directly address the problem of hypoperfusion and provide therapeutic improvement in cerebrovascular circulation. The reduction in brain levels of acetylcholine in patients with vascular dementia is the basis of the cholinergic hypothesis of known onset dysfunctions in adulthood and the rationale for the development of cholinergic therapies in the treatment of this diseases. This study is designed to investigate the combination of two common therapy for $\mathrm{VaD}$ to try to counteract the two pathophysiological mechanisms underlying the $\mathrm{VaD}$ widely discussed in the introduction. The agents chosen for intervention are SDX and choline alphoscerate.

The intake of the two drugs for nine months resulted in a clear improvement in the scores of the parameters taken into consideration (MMSE, ADL and IADL) such as to consider a routine use in the clinical practice of this association. In particular, the greatest results are obtained with the scores of the Mini Mental Examination (+0.7) that improve already from the third month of therapy $(+0.5)$ also because memory functions are more compressed in the $\mathrm{VaD}$.

\section{Conclusions}

As indicated in the introduction, there are essentially two pharmaco-therapeutic strategies for enhancing neurotransmission in vascular dementia: choline alphoscerate and sulodexide. Our hypothesis about the combination of two drugs for VaD may aid for the development of preventive treatment it is realistic. In summary, we found that the combine therapy with choline alphoscerate and sulodexide had a much greater effect than choline alphoscerate alone. The results suggested that the combined formula would be a better therapy to decrease a cognitive impairment for patients with $\mathrm{VaD}$ compared to the mono-therapy with choline alphoscerate. Further research on the molecular mechanism by which SDX plus choline alphoscerate prevents the cognitive impairment of $\mathrm{VaD}$ is required. Large randomized clinical studies are needed to support these observations by performing tests for the purging of executive functions (the only limitation of this study).

\section{References}

1. Hachinski VC, Lassen MA, Marshall J. Multiinfarct dementia. A cause of mental deterioration in elderly. Lancet 1974;2:207-10.

2. Jellinger KA. The enigma of vascular cognitive disorder and vascular dementia. Acta Neuropathol 2007;113:349-88.

3. Vintiers HV, Ellis WG, Zarow C, et al. Neuropathologic substrates of ischemic vascular dementia. J Neuropathol Exp Neurol 2000;60:658-9.

4. Rockwood K, Gauthier S, Erkinjuntti T. Prevention and treatment of vascular dementia: Vascular Cognitive Impairment, London UK: Martin Duniz Ltd; 2002. pp. 587-95.

5. Parnetti L, Mari D, Abate G, et al. Vascular dementia Italian sulodexide study (VA.D.I.S.S.) clinical and biological results. Thrombosis Res 1997;87:225-33.

6. Romain GC. Vascular dementia may be the most common form of dementia in the elderly. J NeurOl Sci 2002;203:7-10.

7. Wiswanathan A, Rocca WA, Tzourio C. Vascular risk factors and dementia: how to move forward? Neurology 2009;72: 368-74.

8. Chui HC, Gonthier R. Natural history of vascular dementia. Alzheimer Dis Assoc Disord 1998;13:S124-30.

9. Richard E, Moll van Charante EP, van Gool WA. Vascular risk factors as treatment target to prevent cognitive decline. J Alzheimers Dis 2012;32:733-40.

10. Roman CG. Vascular dementia: distinguishing characteristics, treatment, and prevention. J Am Geriatr Soc 2003;51: S296-304.

11. Kalaria RN, Erkinjuntti T. Small vessel disease and subcortical vascular dementia. J Clin Neurol 2006;2:1-11;

12. Pantoni L. Cerebral small vessel disease: from pathogenesis and clinical characteristics to therapeutic challenges. Lancet Neurol 2010;9:689-701.

13. Venkat P, Chopp M, Chen J. Models and mechanism of vascular dementia. Exp Neurol 2015;272:97-108.

14. Amenta F, Tayebati SK. Pathways of acetylcholine synthesis, transport and release as targets for treatment of adultonset cognitive dysfunction. Curr Med Chem 2008;15:488-98.

15. Parnetti L, Mignini F, Tomassoni D, et al. Cholinergic precursors in the treatment of cognitive impairment of vascular origin: ineffective approaches or nedle for re-evaluation? J Neurol Sci 2007;257:264-9.

16. Vintiers HV, Ellis WG, Zarow C, et al.
Neuropathologic substrates of ischemic vascular dementia. J Neuropathol Exp Neurol 2000;60:658-9.

17. Court JA, Perry EK, Kalaria KN. Neurotransmitter control of the cerebral vasculature and abnormalities in vascular dementia: vascular cognitive impairment. London UK: Martin Duniz Ltd; 2002. pp.167-85.

18. Traini E, Bramanti V, Amenta F. Choline alphoscerate (alpha-glycerylphosphoryl-choline) an old choline containing phospholipid with a still interesting profile as cognition enhancing agent. Curr Alzheimer Res 2013;10: 1070-9.

19. Bartus RT, Dean RL, Beer B, et al. The cholinergic hypothesis of geriatric memory dysfunction. Science 1982; 217:408-17.

20. Parnetti L. Amenta F, Gallai F. Choline alphoscerate in cognitive decline and in acute cerebrovascular disease: an analysis of published clinical data. Mechan Ageing Develop 2001;122:2041-55.

21. Di Perri R, Coppola G, Ambrosio LA, et al. A multicentre trial to evaluate the efficacy and tollerability of glycerylphosphoryl-choline versus cytidine diphosphocholine in patients with vascular dementia. J Int Med Res 1991;19: 330-41.

22. Abbiati G, Fossati T, Lachmann G, et al. Absorption, tissue distribution and excretion of radiolabeled compounds in rats after administration of (14C)-Lalpha-glyceryl-phosphorylcholine. Eur J Drug Metab Pharmacokinet 1993;18: 173-80.

23. Vezzetti V, Bettini R. Valutazione clinica e strumentale dell'effetto della colina alfoscerato sul decadimento cerebrale. Presse Med 1992;5:141-4.

24. Erkinjuntti T, Romàn GC, Gauthier S. et al. Emerging therapies for vascular dementia and vascular cognitive impairment. Stroke 2004;35:1010-17;

25. Amenta F, Carotenuto A, Fasanaro G, et al. Studio sull'effetto dell'associazione tra l'inibitore delle colinesterasi donepezil e il precursore colinergico colina alfoscerato sui sintomi della malattia di Alzheimer con danno vascolare associato (ASCOMALVA). Primi risultati. Giorn Ital Geriatr 2011;LIX: 89-98.

26. Barkhof F. Guidelines for brain imaging in vascular dementia clinical trials. Int Psychogeriatr 2003;1:273-6.

27. Mattana P, Mannello F, Ferrari P, et al. Vascular pathologies and inflammation: the antinflammatory properties of sulodexide. J Vasc Endovasc 2012; 19:1-7.

28. Roman GC, Tatemichi TK, Erkinjutti T, 
et al. Vascular dementia: diagnostic criteria for research studies. Report of the NINDS-AIREN international workshop. Neurology 1993;43:250-60.

29. Chui HC, Victoroff JI, Margolin D, et al. Criteria for the diagnosis of ischemic vascular dementia proposed by the State of California Alzheimer's Disease Diagnostic and Treatment Centers. Neurology 1992;428:473-80. 